\title{
Cells size themselves up
}

Cells - especially those in developing systems - are under a constant barrage of information from internal and external sources. Two studies highlight the influence of the physical parameters of cells, such as the volume of cells or their compartments, on gene expression patterns and developmental outcomes.

Almost all animals grow by increasing the number of cells and keeping cells of a constant size. Caenorhabditis elegans is peculiar, as most of its tissues expand by growing

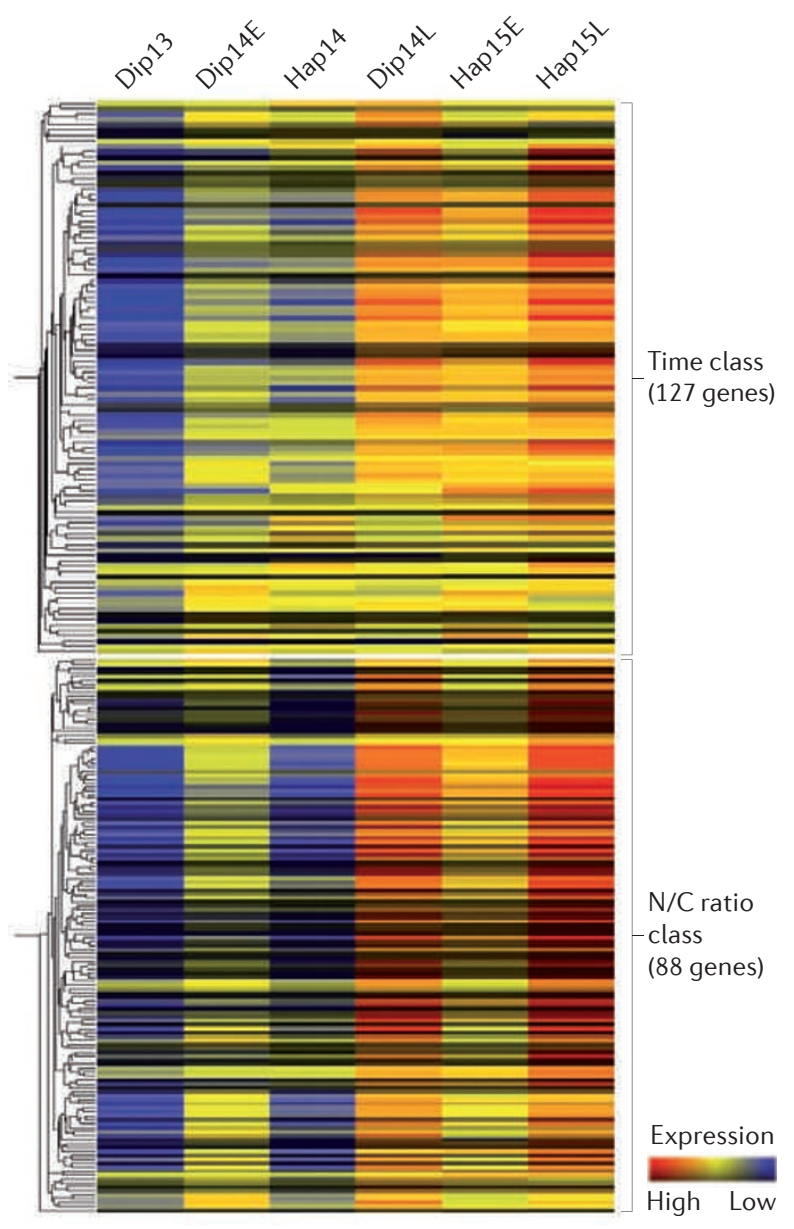

Hierarchical clustering reveals two groups of zygotically supplied transcripts at the Drosophila midblastula transition. Figure courtesy of X. Lu, Princeton University, USA. proportionately more in size than in number, leading to a striking decrease in the surface-to-volume ratio of cells during development. This 'balloon-like' growth suggests hypotheses about the relative abundance of genes that are expressed and about amino acid usage and supply - issues that were examined by Swire and colleagues using transcriptomic and metabolomic data.

By using existing transcriptomic data across five stages of the worm life cycle the authors classified genes as being 'early' or 'late', and assigned them to either the 'cytoplasm' or 'membrane' compartment. This study supports the intuition that the expression of cytoplasmic genes increases with age relative to membrane genes. The growing worm also shifts its amino acid usage; most of the shift is accounted for the by the greater emphasis on producing cytoplasmic proteins (which, for example, use fewer hydrophobic residues). The pool of available amino acids also changes, shifting to match consumption requirements.

Although worms are fairly unique in their growth design, skewed growth geometry is seen in many other settings - from syncytial tissues to abnormal (for example, tumorigenic) polyploid cells - in which the metabolic patterns presented here could be tested. Given how prevalent growth phenotypes are, we should also be aware of the confounding that the balloon effect might have more generally on -omic data analysis.

$\mathrm{Lu}$ and colleagues examined a similar question in Drosophila melanogaster, which, in common with many animals, goes through a shift from a maternal to a zygotic expression pattern during embryogenesis. This midblastula transition (MBT) is also associated with a pause in the cell cycle (cell cycle slowing, CCS) but how might these various events be coordinated?

The authors used chromosome manipulation - to vary the chromosome content compared with wild type - to investigate how the organism might coordinate CCS with zygotic gene activation. The start of CCS does not depend on any specific region of the genome but is triggered by cells reaching a nuclearto-cytoplasmic volume (N/C) ratio of $>70 \%$ of the amount normally present in the embryo at that stage. Of the 290 zygotic genes examined, 127 were activated at the MBT at a certain interval from fertilization that is, they follow an intrinsic timing mechanism (see the figure, top panel); however, a set of $\sim 90$ zygotic genes are activated in response to the $70 \% \mathrm{~N} / \mathrm{C}$ ratio (lower panel). The fact that such genes are activated at the same time and in the same cells that are undergoing CCS argues that the two events are tightly correlated, and that cells are responding to the $\mathrm{N} / \mathrm{C}$ ratio through a common sensing mechanism.

The two papers have highlighted a compelling coordination of cellular and molecular events in two developing organisms. How tightly these coordinated events are mechanistically linked remains to be investigated, as do other puzzling questions, such as the relative timing of sensing and response.

Tanita Casci

ORIGINAL RESEARCH PAPERS Swire, J. et al. The cellular geometry of growth drives the amino acid economy of Caenorhabditis elegans. Proc. R. Soc. Lond. B 13 May 2009 (doi:10.1098/ rspb.2009.0354) | Lu, X. et al. Coupling of zygotic transcription to mitotic control at the Drosophila mid-blastula transition. Development 136, 2101-2110 (2009) 